\title{
COMPLEX TIBIAL PLATEAU FRACTURES TREATED WITH A HYBRID EXTERNAL FIXATOR - LONG TERM RESULTS
}

\author{
Y. Andonov \\ Department of Orthopedics and Traumatology, UMHAT Kaneff AD - Ruse, Bulgaria
}

\begin{abstract}
Introduction: High energy tibial plateau fractures represent a challenge because of the combined bone and soft tissue injury. The iatrogenic impact can further increase the likelihood for crippling complications. Less invasive treatment protocols have been developed to improve the results. Objective: We present the long-term results of limited internal fixation combined with a small wire external fixator. Patients and Methods: Fourteen patients were followed for at least 10 years. Initial and final functional results, complications and presence of joint degeneration were registered according to validated rating scores. Results: Of all patients included in the study, thirteen showed an excellent initial rating and one had an average result at baseline. 10 years later, eight patients still showed excellent grading results, four - good ones, one - average and one - poor results. Ten knees (71\%) had osteoarthritis. Postphlebitic syndrome was diagnosed in two cases. Three knees showed medio-lateral instability, one showed a varus and one - a valgus deformity. Knee arthroplasty was performed in one case. Conclusion: The limited internal fixation combined with a hybrid ex fix can be considered as an alternative to the ORIF, especially when the soft tissues are severely compromised. Despite the inevitable joint degeneration, the associated overall morbidity is low and the long term function is quite satisfactory.
\end{abstract}

Key words: tibial plateau frature, hybrid external fixator, long term results

Corresponding author: Yordan Andonov, UMHAT KANEF AD, 2, Nezavisimost Str., Bg - 7000 Ruse, e-mail: andonov@doctor.bg, http://orcid.org/0000-0002-0186-0411

RECEIVED: 6 February 2021; ACCEPTED: 18 April 2021

\section{INTRODUCTION}

T he complex tibial plateau fractures are the result of a high energy trauma. They are often complicated by significant soft tissue damage. This makes them potentially debilitating on the long term [1]. In contrast to the common perception, it is the instability and the varus malunion, that are detrimental to the overall functional result, not the moderate joint incongruence [2]. This finding may justify a less invasive treatment strategy aiming at diminishing the iatrogenic trauma at the expense of a suboptimal joint reduction $[3,4,5]$. The combination of a limited osteosynthesis, protected by a hybrid external fixator is such a method. We chose this method in the treatment of 26 complex tibial plateau fractures and are herein reporting the long-term results of 14 of them.

\section{MATERIALS AND METHODS}

Twenty-six complex tibial plateau fractures were treated from February 2004 and July 2007 according to a standardized protocol that included limited internal fixation protected by a small wire external fixator. 
Fourteen of the original cases were followed for a period of minimum 10 years. Fractures were classified according to the AO classification and all were of the C type (table 1) [6].

Table 1. Fracture types

\begin{tabular}{|c|c|c|c|}
\hline AO/ASIF & $41 \mathrm{C} 1$ & $41 \mathrm{C} 2$ & $41 \mathrm{C} 3$ \\
\hline $\mathrm{N}$ & 5 & 4 & 5 \\
\hline
\end{tabular}

Ten of the patients were male $(\mathrm{N}=10)$, the remaining - female $(N=4)$. The average age was 58 years. All fractures showed varying degrees of initial soft tissue damage, one of them being open.

We performed closed or limited open reduction and fixed the articular fragments percutaneously by a lag screws. The hybrid external fixator protected the reduction and allowed precise axial and rotation apposition, while allowing early movement of the knee. Bone grafting was done in 5 cases. The results were graded according to the criteria of the Knee Society Clinical Rating Score [7]. This particular scale was widely used in clinical studies $[8,9,10]$. The maximum of one hundred points is distributed in 2 categories: objective (pain, stability and ROM) and functional (walking, climbing) assessment. Results were assessed according to the Watson's criteria [11]. A total sum of over $85 \%$ was considered excellent, $84 \%-70 \%$ as good, $69 \%-60 \%$ as average and results below $60 \%$ as poor. Joint degeneration was assessed according to Kellgren $и$ Lawrence [12]. The osteoarthritis was classified in 4 stages: Stage 1 - minimal joint narrowing with initial bone spur formation; Stage 2 - evident joint narrowing and bone spur formation; Stage 3 - pronounced narrowing and bone spur formation, combined with initial subchondral sclerosis and bone deformity; and Stage 4 - an obliterated joint space, heavy bone spur formation and bone deformity.

\section{RESULTS}

Of all patients included in the study, thirteen received an excellent initial rating and one had an average score. The mean initial objective rating attained 93,8 (82-98) points, the average functional rating was 79,4 (45-100). The average initial ROM was $117,2^{\circ}$ (105120). 10 years later, eight patients still received an excellent rating, four had good ratings, one had an average and one had a poor result (figure 1).

Ten knees $(71 \%)$ showed evidence of osteoarthritis, that was rated as stage 2 in four cases, stage 3 in five cases and stage 4 in one. The functional results of that subgroup of patients are presented in figure 3.

The worst complication was a post-phlebitic syndrome in two cases. It correlated with the worst functional results.

Three knees showed medio-lateral instability, one had a varus and one had a valgus deformity.

Knee arthroplasty was done in one case.

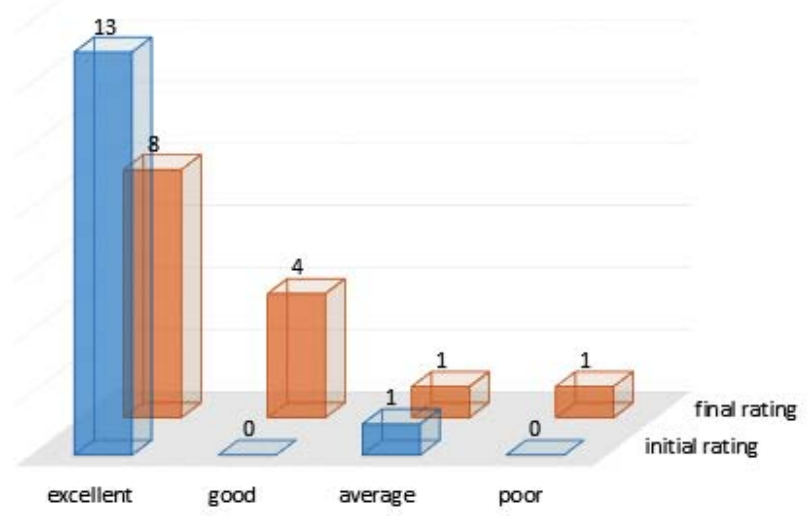

Fig. 1. Comparison between the initial and late functional results

\section{DISCUSSION}

The optimal treatment of the tibial plateau fractures is still a subject for debate. The complexity of these fractures precludes one universal approach [13].

In an already classic study Lansinger followed patients with plateau fractures for an extended 20-year period [14]. The author couldn't find clear connection between the articular depression and the subsequent arthritic degeneration. On the contrary, the residual joint instability was inevitably worsening the functional result [14]. Parkkinen et al. followed 73 patients with type B3 plateau fractures for an average of 53 months. They concluded that a valgus deformity over $5^{\circ}$ as well as articular depression over $2 \mathrm{~mm}$ could result in a more pronounced degeneration not affecting the functional result [15].

The long term follow up of our patients showed a moderate functional deterioration. Most of the knees had arthritic changes $(71 \%)$. Of those only $14 \%$ had lower functional ratings. The major negative prognostic factor in our patients appeared to be the initial thrombophlebitis. Both patients with that complication had long term swelling of their legs and a worse function. Therefore long-term antithrombotic prophylaxis was implemented. 

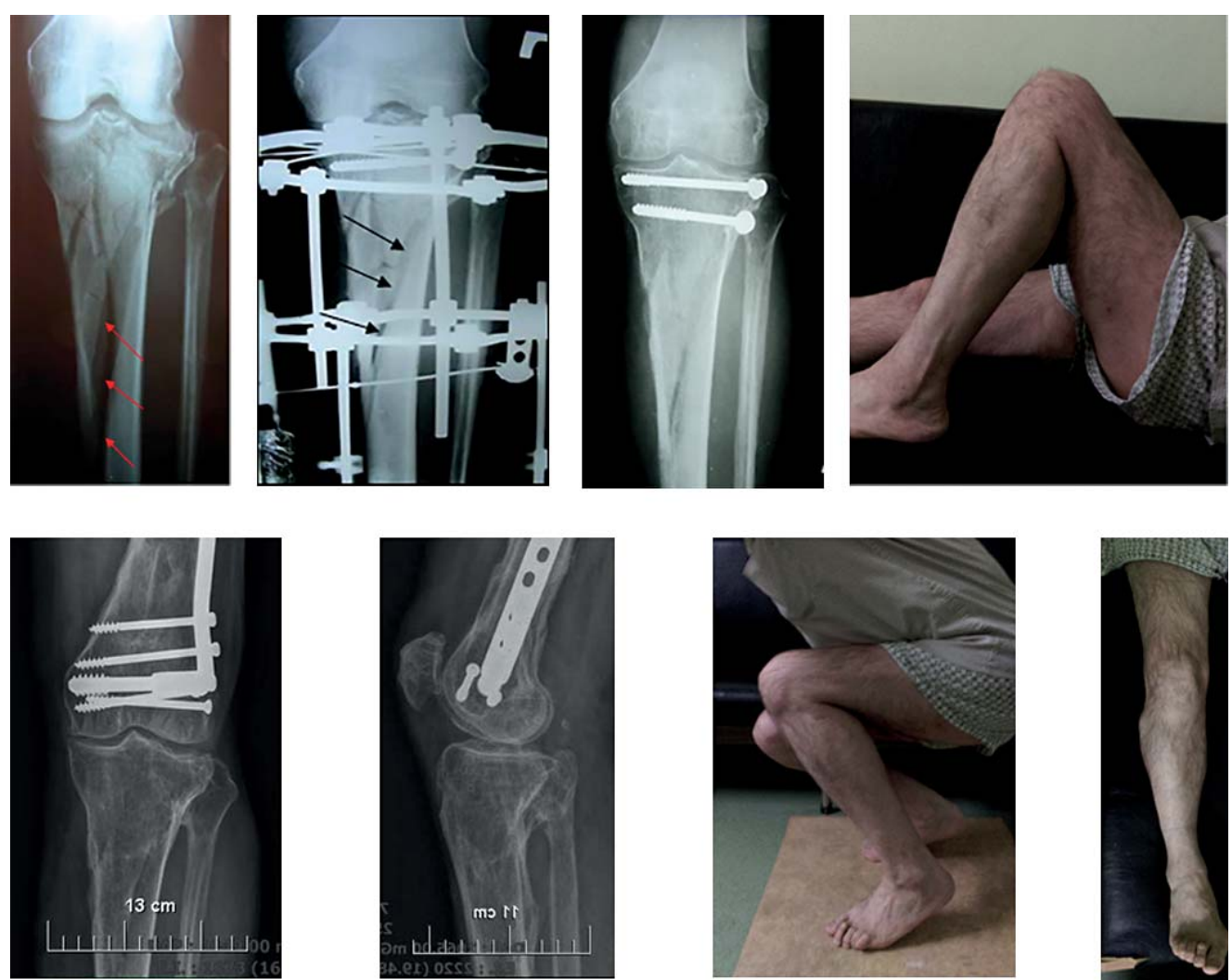

Fig. 2. Patient with a type $41 \mathrm{C} 3.3$ fracture after a vehicle accedent. Unrelated distal femoral fracture 8 years later. Grade 2 osteoarthritic degeneration and an excellent functional result at 11 years after the initial trauma

We were unable to find a correlation between the osteoarthric degeneration and the functional state of the affected patients (figure 2, 3). Most of them were fulfilling their everyday duties and achieved good or excellent ratings.

Polat et al. had followed 52 patients with types B and $C$ plateau fractures for a period of 7 years [16]. Similarly, they didn't find any correlation between the arthritic changes and the functional result [16].
Of the initial 26 fractures included in our study, one only needed arthroplasty (2.3\%). This is in contrast with the general tendency. Dreumel reported that $7,3 \%$ of his patients with plateau fractures had received knee replacement [17]. Timmers published an even larger percentage - 22\% [18]. Wassestein defined the probability for this procedure to be as high as $7,3 \%$ for a 10 -year post-fracture period [19]. The complex fractures of older patients were found to be more likely to need salvage surgery. The treatment

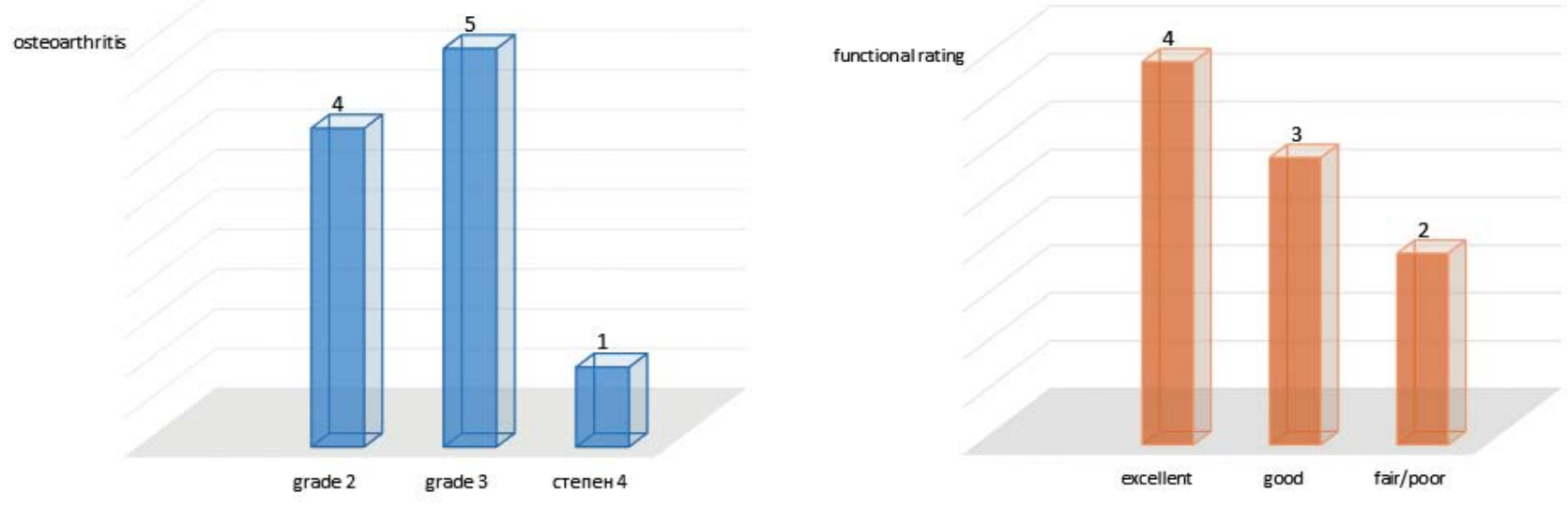

Fig. 3. Functional results of the patients with osteoarthritis 
method in all of the studies was open reduction and plate fixation. In a long term follow up of 83 skiing related plateau fractures, Baumlein et al. reported a lower percentage of arthroplasties $-2,4 \%$, similar to our findings [20]. However, we had less energy skiing fractures which may explain our results.

\section{CONCLUSION}

The current study presents the long term results of a patient series treated according to a novel approach. The less invasive osteosynthesis combined with a hybrid fixator offers an effective solution for the challenging complex tibial plateau fractures. We perceive it is a real alternative to the ORIF, especially when the soft tissues are severely compromised. Despite the inevitable joint degeneration, the associated overall morbidity is low and the long term function quite satisfactory for the patients.

Disclosure summary: The authors have nothing to disclose.

\section{REFERENCES}

1. Ali AM, El-Shafie M, Willett KM. Failure of fixation of tibial plateau fractures. J Orthop Trauma. 2002;16(5):323-9.

2. Honkonen SE. Indications for surgical treatment of tibial condyle fractures. Clin Orthop. 1994;302:199-205.

3. Cole PA, Zlowodzki M, Kregor PJ. Treatment of proximal tibia fractures using the less invasive stabilization system: surgical experience and early clinical results in 77 fractures. J Orthop Trauma. 2004;18(8):528-35.

4. Egol KA, Tejwani NC, Capla EL et al. Staged management of high-energy proximal tibia fractures (OTA types 41): the results of a prospective, standardized protocol. J Orthop Trauma. 2005;19(7):448-55.

5. Raikin S, Froimson MI. Combined limited internal fixation with circular frame external fixation of intra-articular tibial fractures. Orthopedics. 1999;22(11):1019-25.

6. Muëller ME, Nazarian S, Coch P, Schatzker J. A Comprehensive Classification of Long Bones. Berlin, Springer-Verlag, 1990, 170-179.
7. Insall JN, Dorr LD, Scott RD, Scott WN. Rationale of the Knee Society clinical rating system. Clin Orthop Relat Res. 1989;(248):13-4.

8. Kulkarni GS, Kulkarni MG, Kulkarni SG et al. Surgical Treatment of Tibial Plateau Fractures. Orthop Traumatol 2001;9: 263-72.

9. Kumar A, Whittle AP. Treatment of complex (Schatzker type VI) fractures of the tibial plateau with circular wire external fixation: retrospective case review. J Orthop Trauma 2000;14:339-44.

10. Mikulak SA, Gold SM, Zinar DM. Small wire external fixation of high-energy tibial plateau fractures. Clin Orthop Relat Res. 1998;356:230-8.

11. Watson JT, Coufal C. Treatment of complex lateral plateau fractures using Ilizarov techniques. Clin Orthop Relat Res. 1998;(353):97-106.

12. Kellgren JH, Lawrence JS. Radiological assessment of osteoarthrosis. Ann Rheum Dis. 1957;16:494-502.

13. McNamara I, Smith T, Shepherd K et al. Surgical Fixation Methods for Tibial Plateau Fractures Cochrane Database Syst Rev. 2015 Sep 15;(9):CD009679. DOI: 10.1002/14651858. CD009679.pub2

14. Lansinger O, Bergman B, Korner L, Andersson GB. Tibial condylar fractures: a twenty year follow-up. J Bone Joint Surg Am. 1986;68:13-9.

15. Parkkinen M, Madanat R, Mustonen A et al. Factors Predicting the Development of Early Osteoarthritis Following Lateral Tibial Plateau Fractures: Mid-Term Clinical and Radiographic Outcomes of 73 Operatively Treated Patients. Scand J Surg. 2014;103(4):256-62..

16. Polat B, Gurpinar T, Polat A, Ozturkmen Y. Factors Influencing the Functional Outcomes of Tibia Plateau Fractures After Surgical Fixation. Niger J Clin Pract. 2019;22(12):1715-1721.

17. Van Dreumel RLM, van Wunnik BPW, Janssen L et al. Mid- to long-term functional outcome after open reduction and internal fixation of tibial plateau fractures. Injury Int J Care Injured 2015;46:1608-1612.

18. Timmers TK, van der Ven DJC, de Vries LS, van Olden GDJ. Functional outcome after tibial plateau fracture osteosynthesis: a mean follow-up of 6 years. Knee 2014;21(6):1210-1215.

19. Wasserstein D, Henry P, Paterson JM et al. Risk of total knee arthroplasty after operatively treated tibial plateau fracture: a matched-population-based cohort study. J Bone Joint Surg Am. 2014;96(2):144-150.

20. Bäumlein M, Hanke A, Gueorguiev B et al. Long-term Outcome After Surgical Treatment of Intra-Articular Tibial Plateau Fractures in Skiers. Arch Orthop Trauma Surg. 2019;139(7):951-959. 